\title{
On the strong influence of molecular interactions over large distances
}

\author{
Andreas Pfennig ${ }^{\mathrm{a}}$ \\ Department of Chemical Engineering, Université de Liège, 4000 Liège, Belgium
}

Received 29 April 2017 / Received in final form 8 December 2017

Published online 8 March 2018

(C) The Author(s) 2018. This article is published with open access at Springerlink.com

\begin{abstract}
Molecular-dynamics simulations of liquid water show deterministic chaos, i.e. an intentionally introduced molecular position shift of an individual molecule increases exponentially by a factor of 10 in $0.23 \mathrm{ps}$. This is a Lyaponov instability. As soon as it reaches molecular scale, the direction of the resulting shift in molecular motions is unpredictable. The influence of any individual distant particle on an observed molecule will be minute, but the effect will quickly increase to molecular scale and beyond due to this exponential growth. Consequently, any individual particle in the universe will affect the behavior of any molecule within at most 33 ps after the interaction reaches it. A larger distance of the faraway particle does not decrease the influence on an observed molecule, but the effect reaches molecular scale only some ps later. Thus in evaluating the interactions, nearby and faraway molecules have to be equally accounted for. The consequences of this quickly reacting network of interactions on universal scale are fundamental. Even in a strictly deterministic view, molecular behavior is principally unpredictable, and thus has to be regarded random. Corresponding statements apply for any particles interacting. This result leads to a fundamental rethinking of the structure of interactions of molecules and particles as well as the behavior of reality.
\end{abstract}

\section{Introduction}

Molecular behavior is determined by thermal motion of the molecules and the intermolecular forces acting between them. The intermolecular forces strongly decay with decreasing distance between the molecules, so that usually in molecular simulations only the interactions within some molecular diameters are taken into account (e.g. for general aspects [1], or for specific simulations e.g. [2-4]). The effect of interactions with molecules farther away is accounted for via long-range corrections applied to the evaluated thermodynamic functions. Thus, it is assumed that the effect of faraway molecules can be smeared out in time and space.

While this may hold for the averaged thermodynamic functions, it does not hold for the motion of individual molecules, which will behave according to deterministic chaos. As a consequence, the resulting picture of molecular motion and interactions may be misleading. To quantify the influences and to show the resulting fundamental implications, two steps have been taken.

In the first step, the behavior of a molecular system is characterized via molecular-dynamics simulations. It is quantified how strongly the system is governed by deterministic-chaotic behavior.

\footnotetext{
${ }^{\text {a }}$ e-mail: andreas.pfennig@uliege.be
}

That deterministic chaos induces limitations to predictability has often been stated, typically referring to a practical inability to predict system behavior over extended periods of time. As a characteristic example Yin and Herfel [5] write: "... in order to accurately predict even the medium-term behaviour of such a system requires measuring the initial conditions with infinite accuracy. Thus, even though chaotic systems are governed by deterministic dynamic laws, predicting their mediumterm trajectories is in principle impossible." Also Lorenz [6] is frequently cited, after whom the Butterfly Effect is named, who asked: "Does the Flap of a Butterfly's Wings in Brazil Set off a Tornado in Texas". He assumes that the effect of the butterfly wing on weather would only appear in "sufficiently distant future", and concluded that possibly the effect on predictability would be dramatic only after significant time: "The hopes for predicting two weeks or more in advance are thus greatly diminished". Bishop [7] states: "So even if the disturbance of the measurement technique could be eliminated, the observers would still have to make a complete accounting for all the effects just mentioned (the position and motion of every body, every force, etc. in the universe) in order to make a perfect measurement of the chaotic pendulum's position for use in a prediction task. This is a large, though finite, amount of information needed for a full accounting of an accurate measurement of the pendulum's position and certainly qualifies at least as in practice impossible." Also Bishop 
appears to believe that at least in principle it could be possible to predict the behavior of a chaotic pendulum, i.e. a deterministically chaotic system. From these and similar statements it appears to be often expected that at least in principle predictions for deterministically chaotic systems over some "medium-term" are possible.

Crutchfield [8] on the other hand realizes fundamental limitations, because from information loss he theorizes that in a billiard game with the energy being conserved and the billiard balls frequently hitting, "The uncertainty caused by the existence or nonexistence of the electron at the edge of the universe leads to total unpredictability in about six minutes."

To quantify this effect in a more realistic system, in the second step of the current work the strong influence of a distant particle interacting with the system characterized in the first step is investigated. It is demonstrated, that the influence is very much faster than apparently previously anticipated. Finally the consequences of these finding for our understanding of behavior on molecular scale are discussed.

\section{Behavior of a molecular system}

In molecular-dynamics simulations a small number of molecules is followed along their trajectories. Such simulations can then be evaluated to answer a wide variety of specific questions. The macroscopic properties of the molecular system are obtained by thermodynamic averaging of the simulation results over a sufficiently large number of molecules and over a sufficiently extended time period. In the evaluation it is generally assumed that beyond the cut-off distance the influence of individual molecules can be neglected and that the accumulated effect of all molecules outside the cut-off radius can be accounted for by adding a long-range correction to the evaluated thermodynamic variables.

Since it is expected that the molecular system shows deterministic chaos, the intensity of this influence shall be quantified. Deterministic behavior implies that all governing equations are strictly defined so that the complete characterization of the starting conditions of the system fully defines its future development. In chaos theory, chaotic behavior is characterized by a small perturbation introduced increasing exponentially. Thus the effect of small positional shifts on the molecular-dynamics simulations is evaluated.

The simulations have been performed for argon [9] with an available own NVE code applying the Gear predictorcorrector algorithm to 4th and 5th order at different conditions and for water with the MDMPOL program [10] from the CCP5 library, which also applies the NVE ensemble, and the TIP4P/2005 intermolecular potential for water [11]. To these programs, some lines were added that allow to introduce the positional shift of a specified molecule at a specified time step and to record the trajectories of the molecules after that shift. In the un-shifted case, the identical code was used but the shift was set to zero. The simulation programs were run with doubleprecision accuracy using the GNU Fortran compiler [12],

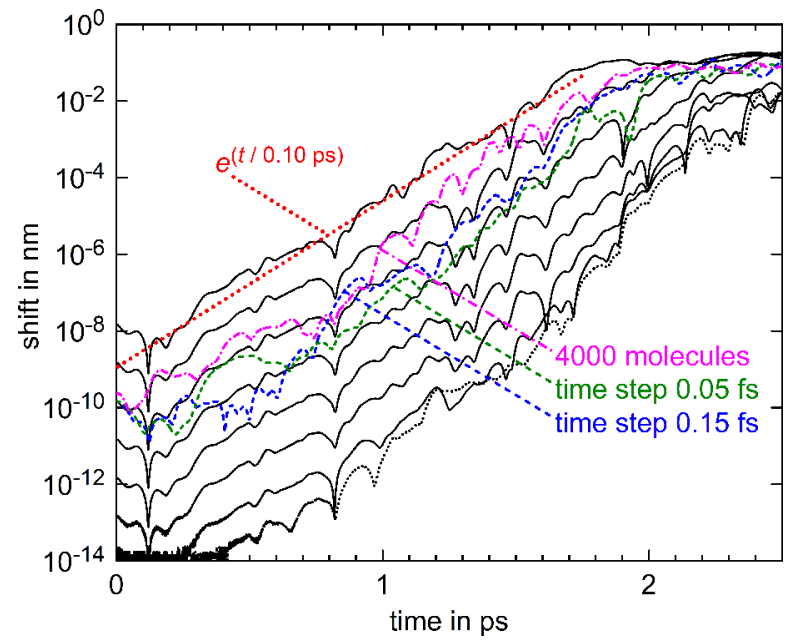

Fig. 1. Initial perturbations of the position of one molecule in a molecular assembly of liquid water at $37^{\circ} \mathrm{C}$ increase exponentially with a Lyapunov time of $0.10 \mathrm{ps}$. The black lines have been obtained with a time step of 0.3 fs for 864 molecules.

i.e. with 8-byte accuracy corresponding roughly to 16 significant digits. The simulations with argon and water lead to comparable results, where the effects are more pronounced with water. Since water is also a molecule of practical relevance, the results are here mainly presented for water.

The simulations were performed for 864 liquid water molecules at $37^{\circ} \mathrm{C}$ and liquid saturation. The time step was $0.3 \mathrm{fs} .5000$ time steps were performed for equilibration starting from a regular starting arrangement with typical random but temperature-scaled velocities of all molecules. During equilibration, temperature corrections were performed every 100 steps. After equilibration had been reached, the position of a single molecule was shifted once in $x$-direction. The trajectory of that molecule was compared between simulations with and without this single shift and the corresponding displacement evaluated.

The results showing the influence of the magnitude of the initial perturbation are depicted in Figure 1. The curves indicate that the deviation of the trajectories increases exponentially as is expected for a deterministically chaotic system. Starting with different initial shifts of the observed particle leads to essentially parallel curves, which show systematic downward peaks. These peaks can be understood from the liquid nature of the system modelled. The observed molecule wiggles within the cage of the nearest neighbors, in which it is bound to meet positions, which are close between shifted and un-shifted molecule, unless a diffusive move in concert with its neighbors occurs, e.g. an exchange of places. Such diffusive moves can be seen in Figure 1 at larger scales, where the curves level off at positional deviations of the order of the particle diameter. Thus on a larger scale the motion as well as the positional shifts induced by the initial shifts are limited by the speed of diffusion, but still continue to increasingly deviate albeit at smaller rate.

At very small initial shifts, as machine precision of 16 decimal digits is approached by the numerical values, 
which are scaled with half the box length, the behavior changes. The curves do not run as parallel any more as for the larger initial shifts. The bumps do not occur at the same times any more. For such small initial shifts, they apparently interact in some specific way with the inaccuracies of the computer, as if the direction of the shift would not be as clearly defined as for larger shifts. If e.g. the initial shift is performed in $y$ - or $z$-direction, the behavior with respect to the observed peaks differs in a similar way from the consequences of a larger shift in $x$-direction.

It is also observed that during roughly the first $0.2 \mathrm{ps}$ the positional shift does not significantly change. This corresponds to some few wiggles of a shifted molecule within the cage of nearest neighbors, which means that these neighbors need to have "experienced" the shift of the perturbed molecule before the deterministically chaotic behavior sets in. This is to be expected, since an initial shift of a single molecule flying in empty space does not increase. Thus for the chaotic behavior to occur, it is required that the neighboring molecules react on the initial perturbation and the perturbed molecule then interacts further with the neighbors which meanwhile have been influenced by the perturbation themselves.

While the exponential increase is expected, it nevertheless has to be ensured that the slope is characteristic for the system itself and not induced by the specifics of the algorithm used. A first indication that this is a system-inherent property is that if it were an aspect of the algorithm, e.g. due to the finite time step used, it would be expected that for a larger magnitude of the initial shift the influence of e.g. the time-step for integration would decrease and as a consequence the slope of the curves should change at a time and spatial scale of which one could assume that it is significantly lower than the particle size. This is not observed anywhere below the diffusional limit, where aside of the statistical variations the slope of all curves is essentially constant independent of time and spatial scale, hinting that the observed exponential increase is characteristic for the system behavior itself. This has also been demonstrated by performing the same simulation with shorter time steps of $0.15 \mathrm{fs}$ and $0.05 \mathrm{fs}$ for one of the simulations, shown as dashed curves in Figure 1, and with a larger system shown as dash-dotted curve, leading to identical slope within the scatter expected due to the statistical nature of the simulations. Finally, simulations for Argon with different time steps, different order of the Gear algorithm applied, and differing system size led to consistent constant slopes as well. Thus, the exponential growth of an initial perturbation in position has to be considered a system property.

Figure 1 also shows a linear fit to one of the curves in the logarithmic plot in its essentially linear range. While this exponential increase is expected for such a system, it is striking, how fast the positional deviation increases. The fitted curve in Figure 1 shows that the positional shift increases by a factor of $e$ in just $0.10 \mathrm{ps}$ in this specific case, which is the so-called Lyapunov time of this deterministically chaotic molecular system. This corresponds to an increase by factor of 10 in just $0.23 \mathrm{ps}$. This means in turn that the starting position of a particle has to be specified

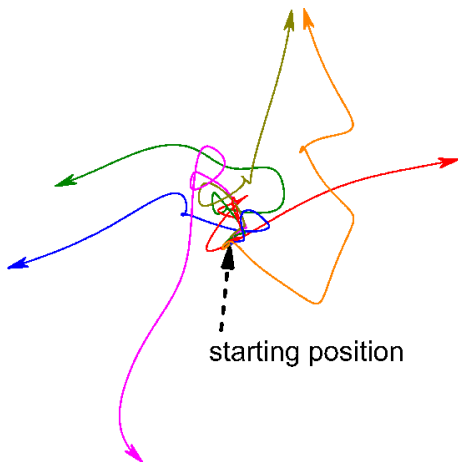

Fig. 2. Successive perturbations of starting positions by $10^{-10}$ molecular diameters lead to randomization of molecular motion as shown in this $x y$-projection of the trajectory of an argon molecule in liquid argon at $120 \mathrm{~K}$.

more accurately by an additional decimal digit for every $0.23 \mathrm{ps}$, for which the trajectory shall be defined with a given accuracy further into the future.

While the starting deviation in position is following a similar spatial trajectory, the final deviation is clearly in arbitrary directions as can be seen in Figure 2, which has been obtained for liquid argon at $120 \mathrm{~K}$ with the starting position of one particle being shifted by multiples of the smallest of these shifts. Thus, the minimal initial shifts appear to randomize the result for any individual particle. This effect is independent of the magnitude of the initial shift. For smaller initial shifts it only takes somewhat longer until a given final deviation in position is reached - scaling with a factor of 10 every $0.23 \mathrm{ps}$.

It should be noted that this behavior is fully expected in principle for such a system. It is known that molecular systems behave as chaotic systems exhibiting the so-called Lyapunov instability describing exactly the observed divergence of molecular trajectories [13-16]. In chaos theory, a wide variety of similar systems has been investigated and discussed [17]. What may be surprising at first sight is the extremely short Lyapunov time.

Thus, the results up to this point represent what is expected in deterministic chaos, namely that any perturbation will increase exponentially - defining the chaotic behavior. As discussed in the introduction, this is usually expressed such that, if the goal were to predict the trajectory farther into the future based on the deterministic equations defining the system, the starting conditions would need to be known with rapidly increasing accuracy [18], which will quickly be practically infeasible, rendering Laplace's demon impossible. Thus, it appears to be a question of practical limitations that prevent accurate long-term predictions for such a system. Expressed this way, i.e. referring to the attempt to describe the behavior of the system, it remains open, if the deterministic chaos on molecular level is only relevant for the description of the system with an appropriate physical model, or if it has some actual consequences on how the system itself behaves in reality. In addition, it appears that the limitation to predict the future of a system based on starting conditions and deterministic model equations is merely a question 
of practicality. If sufficient computer power were available, it appears in principle possible to describe at least the future of a small section of a system including only a small number of molecules, where the effort required of course increases strongly as the time interval to be described increases. This is apparently only a principal possibility. As mentioned, the accuracy required increases by one decimal digit every 0.23 ps. For an accurate prediction over just a second, for the starting values e.g. of each positional coordinate $4.3 \times 10^{12}$ digits would be required. Of course the required computer accuracy increases correspondingly. Thus, while being a possibility in principle, it is not practical even for small systems and any reasonable time of macroscopic relevance.

\section{Influence of an individual distant particle}

To characterize the influence of a distant molecule on an observed molecule in the system considered, the minute initial shift induced by the force between these molecules acting over some time is evaluated as starting point. In principle, the observed molecule experiences all forces acting on it induced by all other molecules around. The forces quickly decrease as distance increases. Neglecting multibody forces for simplicity in these considerations, the overall force acting on the observed molecule is a superposition of all forces induced by all individual other molecules interacting. Since this is a linear superposition, the initial shift induced by any individual interacting molecule in this concert of forces equals that, which is obtained, if its isolated influence is regarded. Thus the initial shift induced by the force of an interacting individual molecule on the observed molecule over some time is independent of all other distant molecules. Thus only the individual interacting molecule is regarded in the following.

This minute initial shift of an observed molecule induced by an individual distant molecule is non-zero. The farther away an influencing molecule is located with respect to the observed molecule, the smaller the interaction will be. Consequently, also the positional shift of the observed molecule will be quickly decreasing as the distance of the second molecule increases. If the observed molecule is then followed for some time, that small shift will be increasing by a factor of ten every $0.23 \mathrm{ps}$, which will quickly lead to recognizable shifts of molecular positions. Here as a realizable shift one molecular diameter is chosen. Such a shift indicates e.g., if the observed molecule is passing some neighboring molecule to the right or to the left, which corresponds to a clear bifurcation of the trajectory.

For the evaluations again liquid water at $37^{\circ} \mathrm{C}$ as in the molecular-dynamics simulation is used with its Lyapunov time of $0.10 \mathrm{ps}$. For the interactions, gravitation is accounted for, which is acting over arbitrary distances in principle. Thus for an interaction of an observed molecule of mass $m_{2}$ with a molecule of mass $m_{1}$ at a distance of $r_{1,2}$ results an interaction force of

$$
F=G \frac{m_{1} m_{2}}{r_{1,2}^{2}}
$$

where $G$ is the gravitational constant of $6.674 \times$ $10^{-11} \mathrm{Nm}^{2} / \mathrm{kg}^{2}$. Here only scalar values are considered for the variables actually representing vectors, since only the absolute value is of relevance. This force leads to an acceleration of the observed molecule according to Newton's law as

$$
F=m_{2} a,
$$

where $a$ is the acceleration experienced by the observed molecule:

$$
a=\frac{\partial^{2} s}{\partial t^{2}}
$$

with $s$ being the position of that molecule and $t$ the time. The starting shift $\Delta s_{0}$ of this molecule induced by the distant particle interacting via gravitation for a short time interval $\Delta t_{\text {interaction }}$ is thus

$$
\Delta s_{0}=\frac{m_{1}}{2 r_{1,2}^{2}} G \Delta t_{\text {interaction }}^{2}
$$

Taking the scaling time $t_{\text {scale }}=0.10 \mathrm{ps}$ into account, which is the Lyapunov time of the regarded system determined above, the molecular displacement will increase by the Lyapunov instability to reach a shift $\Delta s$ after a given time $t$ :

$$
\Delta s=\Delta s_{0} e^{t / t_{\text {scale }}} .
$$

This can be used to answer the question, how long it takes until the interaction force acting for $\Delta t_{\text {interaction }}$ will lead to a shift of the observed particle of the order of one particle diameter, where this "reaction time" depends on the distance of the influencing molecule:

$$
\Delta t_{\text {reaction }}=t_{\text {scale }}\left(\ln \frac{2 \Delta s}{G m_{1}}+2 \ln r_{1,2}-2 \ln \Delta t_{\text {interaction }}\right)
$$

To evaluate equation (6), an appropriate value of $\Delta t_{\text {interaction }}$ has to be chosen. The smaller $\Delta t_{\text {interaction }}$, the smaller is the initial shift and the longer it will take until the final shift will reach molecular scale. Since $\Delta s$ increases exponentially with $t$, which is smaller than the quadratic increase of $\Delta s_{0}$ with $\Delta t_{\text {interaction }}$ for short times, but larger for large $t$, an optimum exists, which minimizes

$$
\Delta t=\Delta t_{\text {interaction }}+\Delta t_{\text {reaction }}
$$

which is the overall time required from a start of an observed molecular interaction to reach $\Delta s$ on molecular scale. With the equations above the minimum $\Delta t$ is reached for

$$
\Delta t_{\text {interaction }}=2 t_{\text {scale }}
$$

with which the following evaluations have been performed. 
For larger distances between two water molecules the influence of gravitation can be regarded as relevant force definitely acting, which is shown in Figure 3. Considering the details of the interaction, it is apparent that the gravitational forces induced by a faraway water molecule acting on the observed water molecule and its neighbors may be quite similar. Thus, rather the relative shift between these neighboring water molecules should be accounted for as starting point of the continual increase of the positional deviation. This is also shown in Figure 3 referred to as "delta interaction" in the legend, where only the difference between two neighboring water molecules is considered, which interact with the water molecule at varied distance.

It is apparent that in both cases it takes at most of the order of some $10 \mathrm{ps}$ until the interaction of an individual faraway molecule induces significant shifts in any observed molecule. Some characteristic distances to our moon, our sun and the Andromeda galaxy have been included as reference. The largest distance shown in Figure 3 actually refers to the size of the observable universe of around $4 \times$ $10^{26} \mathrm{~m}$. Thus, in effect any molecule within the observable universe at any distance from an observed molecule will individually induce a reaction of the observed molecule within very short times. The magnitude of the initial distance deviation between two neighboring water molecules induced by the interaction with a water molecule at the end of the observable universe over $\Delta t_{\text {interaction }}=0.2 \mathrm{ps}$ is of the order of $10^{-150} \mathrm{~m}$, which then increases to molecular scale of roughly $10^{-10} \mathrm{~m}$ in $140 \times 0.23 \mathrm{ps}=32.2 \mathrm{ps}$. It should be mentioned that varying the interaction time $\Delta t_{\text {interaction }}$ between the time step of the molecular simulation of $0.3 \mathrm{fs}$ and the reaction time to a molecule at the end of the observable universe influences the reaction time only marginally, namely between 31.6 and $33.7 \mathrm{ps}$. Thus the choice of interaction time as discussed above appears reasonable. Any other sensible choice will have little influence on the discussion.

Of course, the interaction will travel at most with the speed of light. Thus, effectively the observed water molecule interacts with e.g. a water molecule on Andromeda galaxy, which occurred around 2.5 million years ago, because the distance to Andromeda galaxy corresponds to roughly 2.5 million light years. But when this information on the interaction reaches an observed particle, the reaction will be essentially instantaneous and significant, i.e. have reached molecular dimensions within some 10 ps.

\section{Discussion}

The interactions on the different scales have to be critically put into relation. It may appear physically extreme that such very small starting shifts occur, if individual molecules interact across such large distances. This can be avoided, if larger objects are considered interacting with an observed water molecule. As an example, a star with the mass of our sun at the end of the observable universe interacting with an observed water molecule for $1 \mathrm{~s}$ will lead to a shift of that water molecule by $10^{-34} \mathrm{~m}$. This is a length scale larger than Planck's length, and thus appears

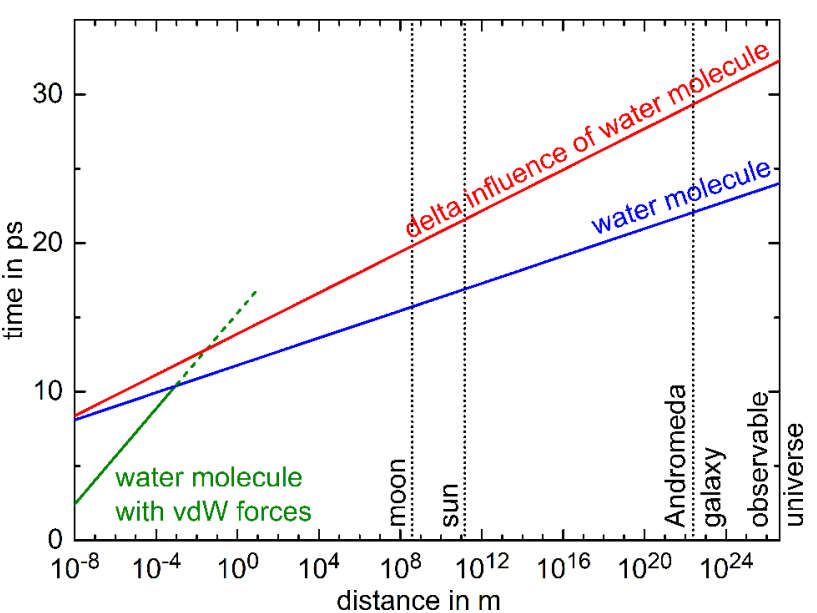

Fig. 3. The influence of a distant water molecule interacting via gravitation on an observed water molecule in liquid water reaches molecular scale after some 10 ps even interacting across the entire observable universe.

to be definitely realistic, which increases to molecular scale within just 5.5 ps. This effect may not appear as dramatic as the interaction between the individual water molecules, but the principal effect remains unaffected.

In addition, the influence of the strong interactions between the molecules in the direct vicinity of the observed water molecule may be considered and the question asked, if those interactions do not dominate the behavior. These interactions are based on physical laws, for which the potential function used in the molecular simulations is only an approximation. In the molecular simulations, the interactions with the close neighbors are accounted for, leading to the Lyapunov time reported. Thus, the existence of the strong interactions between near neighbors is taken care of. These strong interactions of nearby molecules e.g. keep the observed water molecule within the cage of the nearest neighbors in the liquid, which is also responsible for the systematic behavior observed in Figure 1 for shifts below molecular scale. Even though these interactions are much stronger than those with far away molecules, the latter nevertheless have the reported influence, which apparently induce bifurcations of the molecular trajectories as soon as the shift grows to molecular scale. Thus also irrespective of the detailed nature of the interactions between the molecules at short distances the strong influence of far molecules results, if only the Lyapunov instability occurs in molecular systems, which is a system-intrinsic property.

Another aspect to be considered is that in principle quantum mechanics can be applied for a more detailed description of the interactions. Actually gravitation has been regarded here to describe the long-range interactions exactly to avoid such an influence. But of course the intermolecular forces, which lead to the Lyapunov instability, can be described by quantum mechanics. Fortunately, it has already been shown that Lyapunov instability can also be observed in systems described by quantum mechanics [19]. Thus, it can be assumed that the observed effects are general. 


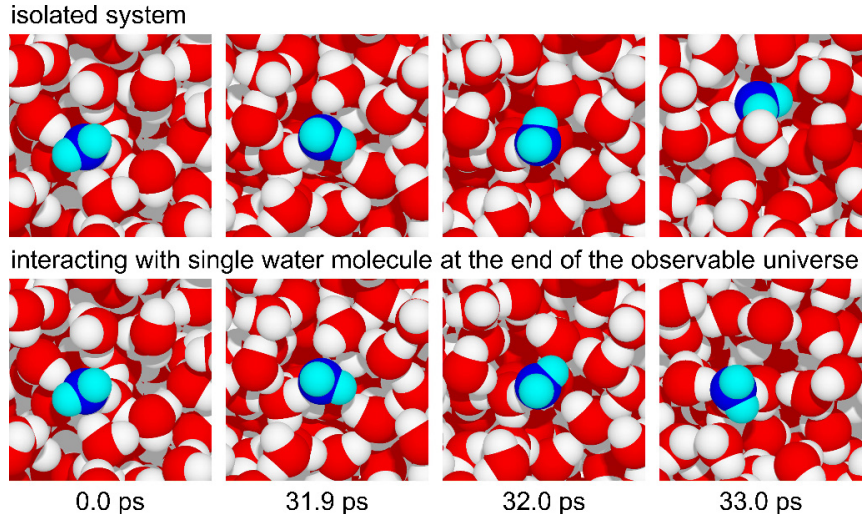

Fig. 4. A single water molecule at the end of the observable universe leads to randomization of a considered ensemble of molecules of liquid water at $37^{\circ} \mathrm{C}$ after only $33 \mathrm{ps}$. One of the observed water molecules is shown in blue for better visualization.

The findings show that every particle at any distance will have an individual influence on every other particle, where even though the forces are extremely small if the distances are large, they quickly lead to shifts on significant scale, since the magnitude of any shift increases by a factor of 10 in $0.23 \mathrm{ps}$ for water molecules in the current example. The interaction is mutual between all molecules. For liquid argon at $120 \mathrm{~K}$, the corresponding time is significantly larger, around $40 \mathrm{ps}$, presumably because the strong orientation-dependent interactions of water together with its fast rotation decrease the reaction time of the system. Nevertheless, also for argon the increase of a displacement is exponential and fast.

It should be clearly stated that it is not such that the interactions stemming from individual molecules at larger distances have lesser influence than those of nearby molecules. Figure 1 shows that any small shift will rapidly grow until it reaches molecular scale. Only the reaction will become "visible" at slightly later times for weaker interactions. This has been demonstrated by corresponding molecular simulations as depicted in Figure 4, where one water molecule in the considered ensemble is shown in blue for better visualization. It has been assumed that at $t=0 \mathrm{~s}$ an interaction with a single water molecule at the end of the observable universe occurs for the bottom simulation shown, whereas it does not occur for the simulation shown above. Of course, the resulting small shift in relative position of the neighbors cannot simply be added to the molecular coordinates in the simulation due to the limited computer accuracy. The shift has thus been evaluated separately and treated according to equation (5). As soon as the shift reached $10^{-12}$ of the scaling length of the simulation, i.e. clearly exceeded computer accuracy, it was added in random direction to the molecular positions. The resulting behavior is shown in Figure 4, where it is seen that $31.9 \mathrm{ps}$ after the interaction, the perturbed and unperturbed systems show only minute differences on the scale considered, which until $32.0 \mathrm{ps}$ increase to become clearly visible and lead to randomization in the sense described above after $33.0 \mathrm{ps}$.
To understand this behavior, the force surface of the resulting gravitational and any other forces acting on any observed particle can be viewed as having a microstructure on an extremely small scale due to the influence of all individual particles in the universe including those essentially arbitrarily far away. Even though this microstructure is so extremely fine, it finally determines the trajectory of the molecules, because it influences their behavior at all of their bifurcations. Since it is practically impossible to resolve this force microstructure, the molecular motion can only be considered as unpredictable. It should be stressed that the decisive microstructure is induced by the interactions between individual molecules. Thus, it cannot be assumed that the interaction forces of faraway molecules can be lumped into an average background interaction. Thus, the influence of faraway molecules will not average out for large distances. Rather each individual interacting particle is determining the course of the molecular trajectories at the bifurcations they pass through.

In contrast to the typical description of the influences due to deterministic chaos resulting in the impossibility to predict molecular trajectories over extended periods of time, the evaluation here directly refers to the behavior of the molecules themselves. This can be expressed more clearly, if the results are summarized as follows:

- Every molecule is constantly "sending" the information of its mass, its position, and possibly other "information" relevant for interaction forces in all directions, which travels at the speed of light.

- Every observed molecule receives this information from all other molecules at any moment in time, which creates the force-microstructure of the resulting overall force realized by the observed molecule, to which the observed molecule reacts with its motion and which influences its behavior at the various bifurcations encountered.

- If any of the other interacting molecules anywhere in the universe would individually not be there or in a slightly different place, the force microstructure experienced by the observed molecule will be different, first leading to a minute shift, which will then due to the Lyapunov instability lead to the observed molecule being at a different place within of the order of some $10 \mathrm{ps}$ at most. After that the displacement increases with the speed of diffusion even to macroscopic scale.

Thus the behavior of the molecules itself is influenced by the deterministic chaos, not just the attempt to predict their future trajectory. Of course referring to the influencing molecule not to exist or to be in a slightly different place is hypothetical, but the described influence is referring to reality, characterizing the quality of the interaction in real world. This effect will apply for any entity carrying a mass or interacting otherwise, i.e. also for elementary particles. All particles in the universe are thus highly interconnected in a quickly reacting network of interactions.

This renders the impossibility of predicting the future of even only a small section of reality based on known 
deterministic physical laws not just a question of practicality. With increasing time interval to be predicted, the horizon of influence, i.e. that spatial horizon for particles, the influence of which needs to be accounted for, is exponentially increasing. For the example of liquid water after only $33 \mathrm{ps}$ all particles in the entire observable universe would need to be considered in the prediction. Since for each particle at least 3 spatial and three velocity coordinates need to be stored and processed, it is fundamentally impossibly to perform such a simulation. Thus, even in a strictly deterministic view molecular trajectories cannot be predicted beyond an extremely short time horizon. Since we thus have no means to compare the deterministic prediction with reality, we can only regard molecular behavior as strictly random.

As a consequence, on the level of individual molecules only a stochastic description is possible. This results from the influence of all particles in the universe. If in an experiment one would succeed to define the exact starting variables of all molecules within an arbitrarily large system, the particles beyond the system boundaries would within at most around $33 \mathrm{ps}$ randomize the molecular behavior inside the system. Thus it has to be realized that the separation between a system and its environment, often considered e.g. in thermodynamics or other areas of physics, is just a virtual separation that in a very fundamental sense cannot be taken to be possible practically, if considered on the scale of individual molecular motion. Practically, isolated systems cannot be realized.

On the other hand, if only two or possibly some few molecules are observed during a limited number of sufficiently isolated interactions, the behavior can be predicted and e.g. from the result the intermolecular potential inferred. Also, if only thermodynamic properties are evaluated, stable values are obtained, because they do not depend on the behavior of the individual molecules but instead resemble ensemble averages.

It should be stressed that the underlying equations are assumed to be deterministically defined, resulting in the trajectories being completely determined as soon as the starting conditions are exactly specified. Thus, here a strictly deterministic viewpoint is taken. Despite our inability to predict the molecular behavior for larger ensembles, the molecules themselves nevertheless behave in a deterministic way, because they do not need to store their position, velocity, etc. with arbitrary accuracy. They simply are at the corresponding exact position, have their velocity, experience the forces, and act according to physical laws without the need to numerically evaluate them. Thus a strictly deterministic behavior, where the evolution of all particles in the entire universe is defined since its start, does not contradict our fundamental inability to in detail predict the behavior.

It should also be mentioned that it is actually to be expected that for real water, which is not just approximated by the TIP4P/2005 intermolecular potential, the reaction time is even shorter than obtained here. Comparison between the Lyapunov times of argon and water indicate that an increased number of degrees of freedom, and their shorter reaction timescale may also reduce the corresponding Lyapunov time. While the timescale for the molecules wiggling within the cage of nearest neighbors is of the order of 10 to $100 \mathrm{fs}$ in the simulations performed, for inter- and intramolecular vibrations of water timescales down to some few fs are reported (see e.g. [20]). Such vibrations may thus further reduce the reaction time of real water molecules. For the variation of atom position within the molecules, the Lyapunov instability may lead to significant shifts already at timescales smaller than the $33 \mathrm{ps}$ found for the model water studied here. Thus, in reality, the some $10 \mathrm{ps}$ reaction time of a system with respect to the farthest molecules is actually to be understood as an upper limit.

Describing the behavior of the observed molecules as passing through bifurcations is actually not exactly depicting the situation. Since at any moment in time every individual molecule will have its decisive influence on an observed molecule, in turn removing any individual molecule would lead to a different direction of the trajectory just a moment later. Thus, the molecules pass through continuous multi-furcations. This emphasizes the statement already made above in relation to Figure 2 that as soon as the divergence of trajectory reaches molecular scale, the direction is fundamentally unpredictable and thus has to be regarded random as well.

The effect will not be confined to molecular scale, but actually will increase to arbitrary scale due to the consequent diffusive motion of the individual molecules. The diffusive shifts above the molecular scale will increase proportionally to the square root of time, scaled with the diffusion coefficient. Thus, the described interactions will influence the position of the molecules also on macroscopic scale, reaching e.g. micrometers after only microseconds.

\section{Conclusions}

The results show that even in a strictly deterministic view the molecular behavior can fundamentally not be predicted for times as short as some pico-seconds. Instead it can only be regarded as random. This means that our picture, which we may typically have of the molecular behavior, where molecules look like their calotte model behaving according to local laws on defined exact trajectories as in the videos obtained from molecular-dynamic simulations, needs modification. Such videos of molecular motion show only one among many options the molecules constantly have. In reality their trajectory is determined by many external influences, which are not accounted for in the simulation. As a result, the actual individual molecular motion can only be described accounting for the stochastic nature induced by the effects presented. Thus, only probabilistic models can describe the real molecular behavior. In turn molecular-dynamics simulations can also only be regarded as producing one of very many options of the real molecular behavior, where implicitly a Monte-Carlo element is always contained.

This resembles the situation encountered in quantum mechanics. Only that on quantum level such nice visualizations of how elementary particles look like are not available. Also an idea on the possibly underlying deterministic governing equations appears missing. In the 
picture presented here, it turns out that any observed particle will undergo continuous minute shifts due to forces induced by the environment. All forces, which do not depend on mass, like Coulomb or van der Waals forces, will lead to initial shifts, which are proportional to the inverse of the mass of the observed particle, as can be seen from equations (2) and (3). On the other hand, gravitation will lead to shifts, which are independent of that mass. As can be seen from Figure 3, the reactions resulting from the van der Waals forces are faster than those induced by gravitation for shorter distances. Thus, effectively these forces combined will lead to an apparent stochastic motion of the molecules, where the diffusion coefficient is to a large extent proportional to the inverse of mass. Interestingly enough, this is a requirement for the stochastic interpretation of the Schrödinger equation [21,22]. Ordinary diffusion coefficients of fluid systems on the other hand typically are proportional to the inverse square root of mass, i.e. they do not show the required behavior. Thus it appears that the Lyapunov instability described here can be the source of the stochastic motion, which is required for the stochastic derivation of Schrödinger's equation, which could not be identified previously [22]. The different dependence of the influence of gravitation may then lead to more complex effects. Corresponding to capillary waves preceding the waves of wavy films, which are induced by nonlinearities and interfacial tension [23], possibly the moving masses can induce similar waves in the elastic fabric of space-time. The elasticity, corresponding to surface tension in liquid films, has already been identified as the reduced Planck constant [24]. This discussion indicates that the Copenhagen interpretation of quantum theory may only be one option. A strictly deterministic view would also produce a clearly stochastic final outcome at the same time avoiding the conceptual challenges related to the observer in the Copenhagen interpretation. The stochastic motion of the particles due to the Lyapunov instabilities induced by the environment thus leads to an ignorance of an observer about the actual position of the particle, where it is actually this continually increasing ignorance, which is described by Schrödinger's equation in this interpretation.

The findings also mean that in situations, where researchers resort to quantum mechanics in order to introduce randomness, like in arguing for freedom of will, the molecular level would fully suffice. Finally, for any deterministically chaotic system corresponding dependencies are expected, of course based on their respective Lyapunov time, if interactions on similar scale apply. Thus, apparently the fundamental interconnectivity on universal scale and the resulting non-locality can have significant implications on our understanding of the structure of reality.

\section{Conflict of interest}

The author declares that there is no conflict of interest regarding the publication of this paper.

The author would like to thank Jadran Vrabec from Paderborn University, Germany, and Peter Schlagheck from the
University of Liège, Belgium, for their supportive discussions, helpful comments, and suggestions. Also I am very grateful for Cédric Gommes' (University of Liège, Belgium) support with the various interpretations of Schrödinger's equation.

Open Access This is an open access article distributed under the terms of the Creative Commons Attribution License (http://creativecommons.org/licenses/by/4.0), which permits unrestricted use, distribution, and reproduction in any medium, provided the original work is properly cited.

\section{References}

1. M.P. Allen, D.J. Tildesley, Computer simulations in liquids (Clarendon Press, Oxford, 1987)

2. M. Thol, G. Rutkai, R. Span, J. Vrabec, R. Lustig, Int. J. Thermophys. 36, 25 (2015)

3. J.C. Palmer, F. Martelli, Y. Liu, R. Car, A.Z. Panagiotopoulos, P.G. Debenedetti, Nature 510, 385 (2014)

4. A. Pfennig, Mol. Simul. 30, 361 (2004)

5. G. Yin, W. Herfel, Constructing post-classical ecosystems ecology, in Philosophy of complex systems, edited by C. Hooker (Elsevier, Amsterdam, 2011)

6. E. Lorenz, The butterfly effect, in The chaos avant-garde: memories of the early days of chaos theory, edited by R. Abraham, Y. Ueda (World Scientific, Singapore, 2000)

7. R.C. Bishop, Erkenntnis 58, 169 (2003)

8. J.P. Crutchfield, Observing complexity and the complexity of observation, in Inside versus outside, edited by $\mathrm{H}$. Atmanspacher, G.J. Dalenoort (Springer, Berlin, 1994)

9. J.A. White, J. Chem. Phys. 111, 9352 (1999)

10. W. Smith, D. Fincham, ftp://ftp.dl.ac.uk/ccp5/MDMPOL (1982)

11. J.L.F. Abascal, C. Vega, J. Chem. Phys. 123, 234505 (2005)

12. GNU, Fortran compiler, https://gcc.gnu.org/ (2016)

13. W.G. Hoover, Nonequilibrium molecular dynamics: the first 25 years. Manuscript UCRL-JC-Preprint CONF9208276-1, in Proceedings of the 18th IUPAP International Conference on Statistical Physics, August 2-8, Berlin, Germany (1992)

14. G. Sutmann, Molecular dynamics - vision and reality, in Computational nanoscience: do it yourself! NIC series, edited by J. Grotendorst, S. Blügel, D. Marx (John von Neumann Institute for Computing, Jülich, 2006), Vol. 31, pp. $159-194$

15. D. Frenkel, arXiv:1211.4440v1 (2012)

16. D.J. Evans, D.J. Searles, Adv. Phys. 51, 1529 (2002)

17. A.C. Scott, The nonlinear universe: chaos, emergence, life (Springer, Berlin, 2007)

18. R.P. Feynman, R.B. Leighton, M. Sands, The Feynman lectures on physics (Addison-Wesley Publishing, Reading, 1963)

19. R.A. Jalabert, H.M. Pastawski, Phys. Rev. Lett. 86, 2490 (2001)

20. K. Ramasesha, L. De Marco, A. Mandal, A. Tokmakoff, Nat. Chem. 5, 935 (2013)

21. E. Nelson, Phys. Rev. 140, 1079 (1966)

22. E. Nelson, J. Phys.: Conf. Ser. 361, 012011 (2012)

23. G.F. Dietze, J. Fluid Mech. 789, 368 (2016)

24. J.W.M. Bush, Annu. Rev. Fluid Mech. 47, 269 (2015) 